Original Research Paper

\title{
About the Light Dimensions
}

\author{
${ }^{1}$ Florian Ion T. Petrescu and ${ }^{2}$ John Kaiser Calautit \\ ${ }^{I}$ Department of Theory of Mechanisms and Robots, Bucharest Polytechnic University, Bucharest, Romania \\ ${ }^{2}$ Department of Mechanical Engineering, University of Sheffield, Sheffield, United Kingdom
}

Article history

Received: 02-26-2016

Revised: 02-27-2016

Accepted: 03-18-2016

Corresponding Author:

Florian Ion T. Petrescu

Department of Theory of

Mechanisms and Robots,

Bucharest Polytechnic

University, Bucharest, Romania

Email: petrescuflorian@yahoo.com

\begin{abstract}
Light is one of the most fascinating something of all the time. This paper aims to develop a tool with which one can determine with great accuracy the dimensions of light. Basically was entered a new relationship through which one may determine the size of a photon depending on the wavelength of light. Instead wavelength of light can be used as variable the speed of light and its frequency. One could determine this new expressions using and the known equations: the total energy of a moving particle and the total energy of a photon, as Einstein bequeathed them to us. It has also been used (for calculating the new relationship) and the kinetic energy of the photon. The new expressions introduced by this paper may be useful in many applications related to light, lasers, etc. This paper is a check point for new "start points".
\end{abstract}

Keywords: Nanoparticles, Light, LASER, Photon, Photon Dimension, Light Dimensions, Light Wavelength, Light Speed, Light Frequency, Photon Size

\section{Introduction}

In general, for determining the size of atomic and subatomic particles their static diameters (ie when the particle is at rest) are used, which is calculated by various approximated methods (Halliday, D. and R. Robert, 1966), (Koberlein, 2015), (Petrescu, 2012), (Wikipedia). These dimensions are of the order of nano, pico or slightly lower size. The real phenomena occur when these particles are in dynamically movement and it is therefore necessary to know the real dimensions of the particles in movement. This paper aims to accomplish this for light. Light is one of the most fascinating elements of all time (said improperly, because the light is not an element; the light has no atoms of any element; the light has only photons). This paper aims to develop a tool with which we can determine with great accuracy the dimensions of light. Basically was entered a new relationship through which one can determine the size of a photon depending on the wavelength of light. Instead wavelength of light can be used as variable the speed of light and its frequency. To determine this new relationship, were used two known equations: the total energy of a moving particle and the total energy of a photon, as Einstein bequeathed them to us. It has also been used (for calculating the new relationship) and the kinetic energy of the photon. The new expressions introduced by this paper may be useful in many applications related to light, lasers, etc.

\section{Materials and Methods}

First one introduces the known Einstein's equation which gives the total energy of an elementary particle on movement (relationship 1), (Halliday, D. and R. Robert, 1966).

$$
E=m \cdot c^{2}
$$

Second one presents the known (Planck-Einstein) equation who gives the total energy of a photon (relationship 2), (Halliday, D. and R. Robert, 1966).

$$
E=h \cdot \gamma
$$

Any elementary moving particle possesses the kinetic energy given by the relationship 3 (composed by two components: the kinetic energy of motion translational and rotational kinetic energy of motion).

And a photon has this total kinetic energy (see the relationship 3). For a photon this total kinetic energy is the same with its total energy, because a photon does not have a rest energy.

$$
E \equiv E_{c}=\frac{1}{2} m \cdot c^{2}+\frac{1}{2} J \cdot \omega^{2}
$$

Mechanical moment of (mass) inertia of a particle (around its axis of rotation) is determined by the relationship 4 . 


$$
J=\frac{2}{5} m \cdot R^{2}
$$

This is the mass moment of inertia of a sphere (see the Fig. 1).

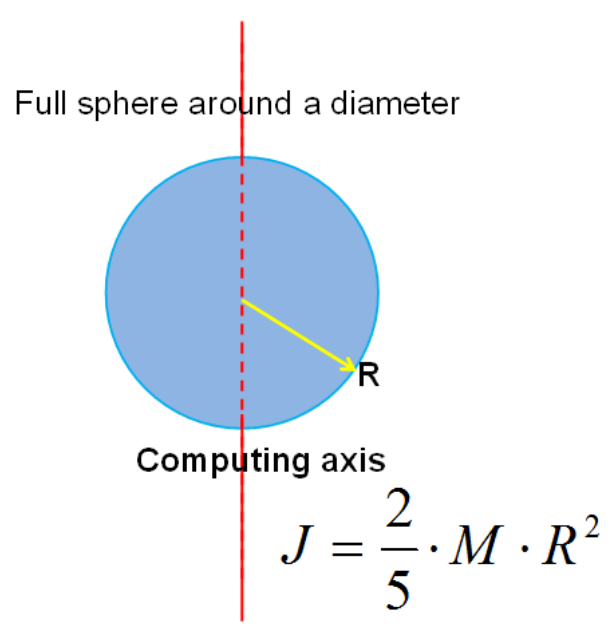

Fig. 1. Mass moment of inertia to a full sphere, determined around a diameter

Using the expression 4 , the relationship 3 takes the form 5 .

$$
E \equiv E_{c}=\frac{1}{2} m \cdot c^{2}+\frac{1}{2} \frac{2}{5} m \cdot R^{2} \cdot \omega^{2}
$$

For the light one can use the relationships 6 to determine the rotational speed of a photon.

$\left\{\begin{array}{l}\omega=2 \pi \cdot \gamma \\ \omega^{2}=4 \pi^{2} \cdot \gamma^{2}\end{array}\right.$

With the help of expressions 6 the formula 5 takes the forms 7 .

$$
\left\{\begin{array}{l}
E \equiv E_{c}=\frac{1}{2} m \cdot c^{2}+\frac{1}{2} \frac{2}{5} m \cdot R^{2} \cdot 4 \cdot \pi^{2} \cdot \gamma^{2} \\
E=\frac{1}{2} m \cdot c^{2}+\frac{1}{2} \frac{8}{5} \cdot \pi^{2} \cdot m \cdot R^{2} \cdot \gamma^{2}
\end{array}\right.
$$

The light frequency can be written in function of the light speed and wavelength using the relationships 8 (Halliday, D. and R. Robert, 1966).

$$
\left\{\begin{array}{l}
\gamma=\frac{c}{\lambda} \\
\gamma^{2}=\frac{c^{2}}{\lambda^{2}}
\end{array}\right.
$$

Using these two expressions (8) the equations 7 take the forms 9 .

$$
\left\{\begin{array}{l}
E=\frac{1}{2} m \cdot c^{2}+\frac{1}{2} \frac{8}{5} m \cdot R^{2} \cdot \pi^{2} \cdot \frac{c^{2}}{\lambda^{2}} \\
E=\frac{1}{2} m \cdot c^{2} \cdot\left[1+\frac{8}{5} \cdot \pi^{2} \cdot R^{2} \cdot \frac{1}{\lambda^{2}}\right]
\end{array}\right.
$$

One can write the mass of one photon (in movement) by expression 10, utilizing the first two equations $(1+2)$ and expression 8 (Halliday, D. and R. Robert, 1966).

$$
m=\frac{h \cdot \gamma}{c^{2}}=\frac{h \cdot c}{\lambda \cdot c^{2}}=\frac{h}{c \cdot \lambda}
$$

Using the expression 10 , the relationships 9 take the forms 11 .

$$
\left\{\begin{array}{l}
E=\frac{1}{2} \frac{h \cdot c^{2}}{c \cdot \lambda} \cdot\left[1+\frac{8}{5} \cdot \pi^{2} \cdot R^{2} \cdot \frac{1}{\lambda^{2}}\right] \\
E=\frac{1}{2} \frac{h \cdot c}{\lambda} \cdot\left[1+\frac{8}{5} \cdot \pi^{2} \cdot R^{2} \cdot \frac{1}{\lambda^{2}}\right]
\end{array}\right.
$$

With the help of the relationships 2 and 8 one obtains the expression 12 .

$E=\frac{h \cdot c}{\lambda}$

Using the expression 12 , the relationships 11 will take the forms 13 .

$$
\left\{\begin{array}{l}
\frac{h \cdot c}{\lambda}=\frac{1}{2} \frac{h \cdot c}{\lambda}\left[1+\frac{8}{5} \cdot \pi^{2} \cdot R^{2} \cdot \frac{1}{\lambda^{2}}\right] \\
2=1+\frac{8 \pi^{2} \cdot R^{2}}{5 \lambda^{2}} \\
1=\frac{8 \pi^{2} \cdot R^{2}}{5 \lambda^{2}} \\
R^{2}=\frac{5 \cdot \lambda^{2}}{8 \cdot \pi^{2}}
\end{array}\right.
$$

From the last expression of relationships 13, one should determine the value of a photon dimension, the photon radius, in function of its wavelength (expression 14).

$$
R=\sqrt{\frac{5}{8}} \cdot \frac{\lambda}{\pi}=\sqrt{\frac{5}{8}} \cdot \frac{1}{\pi} \cdot \lambda
$$

With the expression 8 , the relationship 14 takes the form 15, which shows the radius of a photon in 
function of its velocity (the light speed) and frequency.

$$
R=\sqrt{\frac{5}{8}} \cdot \frac{1}{\pi} \cdot \frac{c}{\gamma}
$$

\section{Results and Discussion}

First, with the expression 14, one can determine the value of a photon dimension, the photon radius, in function of its wavelength.

Using the expression 15 one should determine the radius of a photon in function of its speed (the light speed) and frequency.

For visible light:

$\gamma=1 \mathrm{E}+15[\mathrm{~Hz}], \mathrm{R}_{\text {photon }}=7.54435 \mathrm{E}-08[\mathrm{~m}]=75[\mathrm{~nm}]$.

For radiation $\mathrm{X}$ :

[nm].

$\gamma=1 \mathrm{E}+17 \quad[\mathrm{~Hz}], \quad \mathrm{R}_{\text {photon }}=7.54435 \mathrm{E}-10 \quad[\mathrm{~m}]=0.75$ [nm].

$\gamma=1 \mathrm{E}+20[\mathrm{~Hz}], \mathrm{R}_{\text {photon }}=7.54435 \mathrm{E}-13[\mathrm{~m}]=0.00075$

For Gamma radiation:

$\gamma=1 \mathrm{E}+22 \quad[\mathrm{~Hz}], \quad \mathrm{R}_{\text {photon }}=7.54435 \mathrm{E}-15 \quad[\mathrm{~m}]$ $=0.0000075[\mathrm{~nm}]$.

The results obtained indicate that the relationship is poor. Linear variation is us unexpectedly.

A photon that has very low frequency would mean to increase a lot its diameter.

It feels like something is missing from the theory used. That seems to be just the rest mass of the photon. The equation 3 needs a change, and it should take the form 16 (the addition of translational and rotational kinetic energy and rest energy appears).

$E=\frac{1}{2} m \cdot c^{2}+\frac{1}{2} J \cdot \omega^{2}+m_{0} \cdot c^{2}$

In this case the expression 14 takes the forms 17 and 18 .

$$
\begin{aligned}
& R=\frac{1}{\pi} \cdot \sqrt{\frac{5}{8}} \sqrt{1-2 \cdot \frac{m_{0}}{m}} \cdot \lambda \\
& R=\frac{1}{\pi} \cdot \sqrt{\frac{5}{8}} \sqrt{1-2 \cdot \frac{m_{0} \cdot c \cdot \lambda}{h}} \cdot \lambda
\end{aligned}
$$

Expressions 17-18 may be written and in the forms 19 .

$$
\left\{\begin{array}{l}
\frac{R}{\lambda}=\frac{1}{\pi} \cdot \sqrt{\frac{5}{8}} \sqrt{1-2 \cdot \frac{m_{0}}{m}} \\
\frac{R}{\lambda}=\frac{1}{\pi} \cdot \sqrt{\frac{5}{8}} \sqrt{1-2 \cdot \frac{m_{0} \cdot c \cdot \lambda}{h}}
\end{array}\right.
$$

The rest mass of a photon varies depending on the frequency of the photon. For any frequency the photon rest mass is slightly smaller than half of the moving mass of the photon (see the expressions 20).

$$
\left\{\begin{array}{l}
m_{0}=\frac{1}{2} \cdot m \cdot\left(1-\frac{8 \cdot \pi^{2} \cdot R^{2}}{5 \cdot \lambda^{2}}\right) \\
m_{0}=\frac{1}{2} \cdot \frac{h}{c \cdot \lambda} \cdot\left(1-\frac{8 \cdot \pi^{2} \cdot R^{2}}{5 \cdot \lambda^{2}}\right)
\end{array}\right.
$$

\section{Conclusion} time.

Light is one of the most fascinating something of all

This paper aims to develop a tool with which we can determine with great accuracy the dimensions of light. With the new expressions (19) introduced one can determine the radius of any photon, in function of its wavelength (or light speed and frequency), but only if one may determine first the rest mass of the photon.

The rest mass of a photon varies depending on the frequency of the photon.

For any frequency the photon rest mass is slightly smaller than half of the moving mass of the photon.

Instead wavelength of light can be used as variable the speed of light and its frequency. To determine this new relationship, were used two known equations: the total energy of a moving particle and the total energy of a photon, as Einstein bequeathed them to us. It has also been used (for calculating the new relationship) and the kinetic energy of the photon.

The new expressions introduced by this paper may be useful in many applications related to light, lasers, etc.

It is possible that a photon size to be extremely small, and therefore he was not been revealed until now.

\section{Assumption Used}

1 -Was used the assumption that light frequency is the same with the rotational frequency of the photon.

2-The light has a rest mass. The photon has a rest mass as any other elementary particle. 


\section{Importance}

With the new expressions (19) introduced one can determine the radius of any photon, in function of its wavelength (or light speed and frequency), but only if one may determine first the rest mass of the photon.

\section{An exercise of Imagination}

Using the expressions 19 one presents below some possible values:
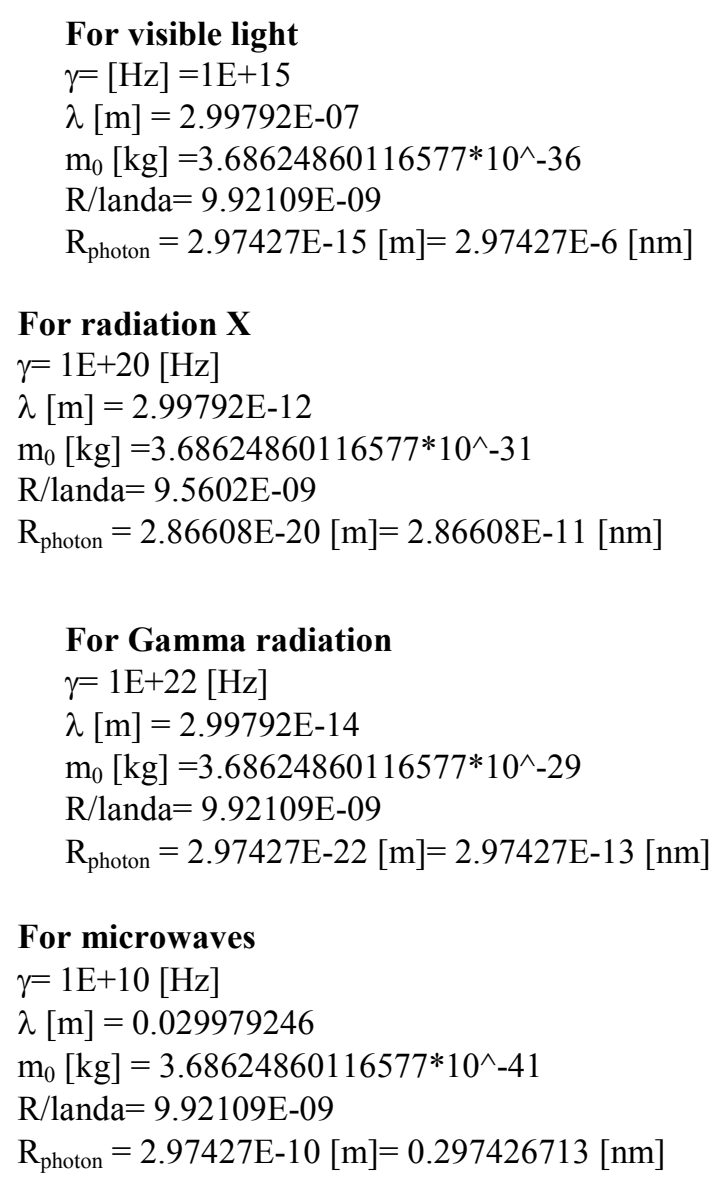

\section{For Radio waves}

$\gamma=1 \mathrm{E}+7[\mathrm{~Hz}]$

$\lambda[\mathrm{m}]=29.9792458$

$\mathrm{m}_{0}[\mathrm{~kg}]=3.68624860116577 * 10^{\wedge}-44$

$\mathrm{R} / \mathrm{landa}=9.92109 \mathrm{E}-09$

$\mathrm{R}_{\text {photon }}=2.97427 \mathrm{E}-07[\mathrm{~m}]=297.4267131[\mathrm{~nm}]$

\section{Answer for Skeptics}

Why radio photons are not visible when they have nearly $300 \mathrm{~nm}$ ? Because they have an extremely small mass (so a very very low density), and are colorless, insipid, odorless. In addition they do not possess even an electric charge. But the main problem is the calculation error due to the limitation of used software.

\section{Software Limitations}

The software used (excel) only works with 14 decimal places. To understand the error introduced by calculation, should we follow the below results.

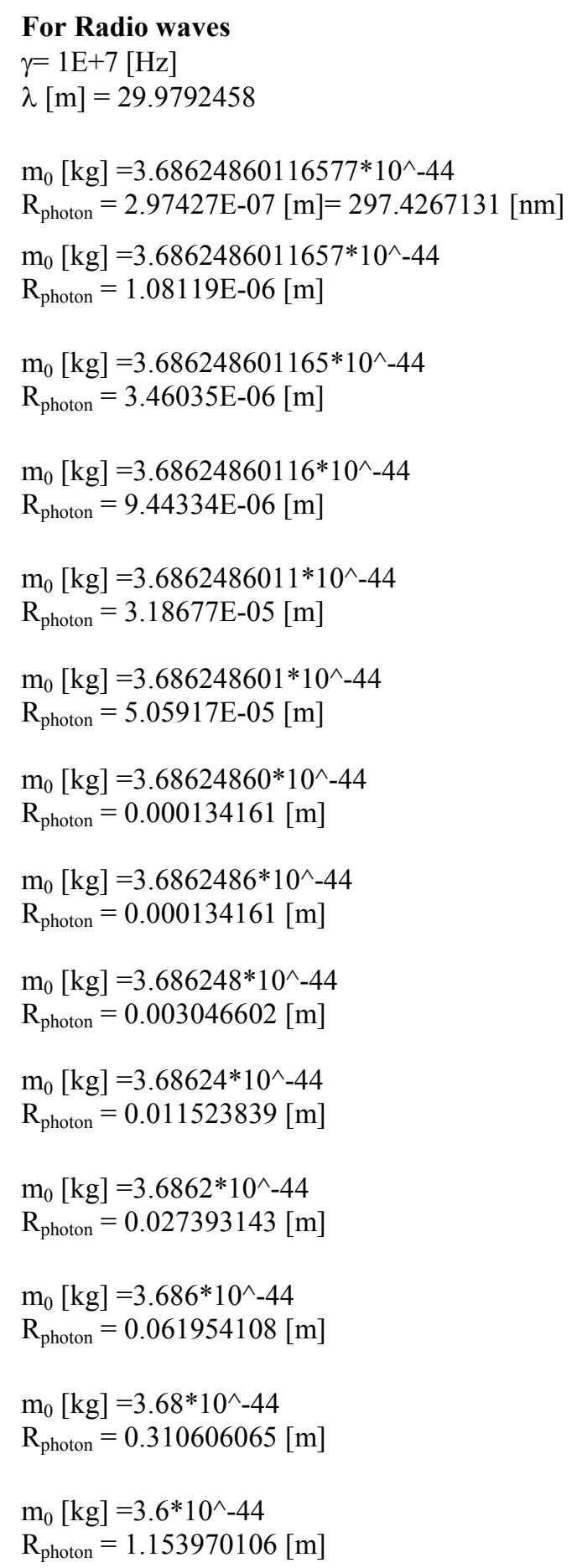


With every two to three decimal places added to mass, the radius decreases about 10 times. If they use in calculation all the 44 decimal necessary, the value of a photon radius (for radio waves) would reach at:

$$
R_{\text {photon }}=2.97427 \mathrm{E}-17[\mathrm{~m}]=2.974267131 \mathrm{E}-8[\mathrm{~nm}]
$$

The value of a photon radius (for microwaves) would reach at:

$R_{\text {photon }}=2.97427 \mathrm{E}-19[\mathrm{~m}]=2.97426713 \mathrm{E}-10[\mathrm{~nm}]$

The value of a photon radius (for Gamma radiation) would reach at:

$R_{\text {photon }}=2.97427 \mathrm{E}-27[\mathrm{~m}]=\mathbf{2 . 9 7 4 2 7 \mathrm { E } - 1 8 [ \mathrm { nm } ]}$

The value of a photon radius (for radiation $\mathrm{X}$ ) would reach at:

$R_{\text {photon }}=2.86608 \mathrm{E}-26[\mathrm{~m}]=2.86608 \mathrm{E}-17[\mathrm{~nm}]$

The value of a photon radius (for visible light) would reach at:

$R_{\text {photon }}=2.97427 \mathrm{E}-23[\mathrm{~m}]=2.97427 \mathrm{E}-14[\mathrm{~nm}]$

\section{Comments}

The mass of a photon increases with its frequency. The radius of a photon decreases when the photon increases its frequency. Even if they have the slightest ray, gamma photons are the most dangerous, with the largest mass and energy. They are the most penetrating.

Can be dangerous and the low-frequency photons, though they have extremely small masses, have much larger dimensions.

\section{Acknowledgement}

This text was acknowledged and appreciated by Assoc. Pro. Taher M. Abu-Lebdeh, North Carolina A and T State Univesity, United States, Samuel P. Kozaitis, Professor and Department Head at Electrical and Computer Engineering, Florida Institute of Technology, United States, and Guanying Chen, Harbin Institute of Technology\&SUNY Buffalo, China.

\section{Author's Contributions}

Florian Ion T. Petrescu: Established the new calculation relationships presented in the paper.

John Kaiser Calautit: Participated in all experiments, coordinated the data-analysis and contributed to the writing of the manuscript and English correction.

\section{Ethics}

This article is original and contains unpublished material. The corresponding author approved the manuscript and confirms that no ethical issues involved.

\section{References}

Halliday, D. and R. Robert, 1966. Physics, Part II. 1st Edn., John Wiley and Sons, Inc., New York.

Koberlein, B., 2015. That's About the Size of It. Retrieved from:

https://briankoberlein.com/2015/04/14/thats-aboutthe-size-of-it/

Petrescu, F.I., 2012. Cold nuclear fusion. plasma physics and fusion technology (S70), INIS 44(16).

Wikipedia, Photon. Retrieved from: https://en.wikipedia.org/wiki/Photon

\section{Nomenclature}

$\mathrm{h}=>$ the Planck constant

$h=6.626070040(81) E-34[J \cdot s]$

$\mathrm{c}=$ the light speed in vacuum

$c=299792458\left[\frac{m}{s}\right]$

$\mathrm{m}_{0}=$ the rest mass of the photon

$\mathrm{m}=$ the mass of the moving photon 